\title{
Simulation of the Flat-parallel Movement of a Bridge Agricultural Unit with an Articulated Frame
}

\author{
Volodymyr Bulgakov1, *Semjons Ivanovs², Volodymyr Volskyi ${ }^{3}$, Volodymyr Kuvachov ${ }^{4}$, Yevhen \\ Ihnatiev $^{4}$ \\ ${ }^{1}$ National University of Life and Environmental Sciences of Ukraine, Ukraine \\ ${ }^{2}$ Latvia University of Life Sciences and Technologies, Latvia \\ ${ }^{3}$ National Scientific Centre "Institute for Agricultural Engineering and Electrification”, Ukraine \\ ${ }^{4}$ Dmytro Motornyi Tavria State Agrotechnological University, Ukraine
}

\begin{abstract}
The work presents foundations of the dynamics of the flat-parallel movement of a bridge agricultural unit in a horizontal with the kinematic method of its control (turning the wheels) by changing the position of one of its parts relative to the other. The analysis of assessment of the degree of impact of the scheme and parameters of the investigated agricultural tool upon its controllability and stability of movement is based on the amplitude and phase frequency characteristics. The mathematical models of the movement of the bridge agricultural unit are presented in a differential and an operator form of recording. Based on mathematical models, the calculated amplitude and phase frequency characteristics of the dynamic control system of the control impact, presented as the angular displacement of its half-frames $\psi$, are constructed for various parameters and operating modes. The constructed mathematical models, amplitude and phase frequency characteristics make it possible to assess the impact of the control parameter of the bridge agricultural unit, as well as its design and other parameters upon the controllability of the movement.
\end{abstract}

Keywords: farming, wide span tractor, pivot, frame, turning, mathematical modelling.

\section{Introduction}

The analysis of scientific works on the state and trends in the development of agricultural mechanisation means is a witness that the widespread use of traditional tractor-combine technologies has created a number of serious problems, the solution of which is impossible within the framework of generally accepted methods of growing cultivated plants (Chamen, 2015; Pogorely, 2008; Zhalnin \& Mufteyev, 2002). According to many scientists in the world, a perspective direction for sustainable development of agriculture is introduction of advanced technologies and technical means, which should include the track system of agriculture (Tullberg, 2013; Bulgakov et al., 2020; Bulgakov et al., 2018). Practical implementation of the basic principles of the track system of agriculture, based on the traditional tractor-combine means of mechanisation of agricultural production, is complicated by certain problems in their use. Some of them should include impossibility of adjustment the parameters of the running systems of serial machine-and-tractor and combine aggregates to the parameters of a constant tramline, the traction properties of the power (energy) equipment with the operating width of the agricultural machines, etc.

According to such scientists as Pedersen $\mathrm{HH}$, Chamen W., Onal I. and others, it is possible to implement this farming strategy in another most perspective radical way through the creation of the socalled "wide span vehicle" (Pedersen, Oudshoorn, \& McPhee, 2016; Onal, 2012; Bulgakov et al., 2017a). Typical world representatives of this kind of bridge agricultural equipment are the "Dowler bridge tractor", BIOTRAC, ASA-Lift WS 9600, an agricultural vehicle of our design and others. The conducted tests of the indicated bridge agricultural means have established that their use is characterised by high potential technical, operational and technological properties. However, their testers, having made a

\footnotetext{
* Corresponding Author's email: semjons@apolo.lv
} 
certain contribution to clarifying the prospects and substantiating the efficiency of the use of the bridge agricultural means, do not always take into account the possibility of practical implementation of their solutions (Pogorely, 2008; Bulgakov et al., 2018). More than that, these researchers have not developed an appropriate methodology for the selection and substantiation of the schemes, parameters and operating modes of the bridge agricultural equipment with an articulated frame during their operation under the conditions of the track system of farming, based on a substantive analysis of the stability and controllability of their working movement along the tracks of a constant tramline.

Mathematical models of functioning of the conventional machine-and-tractor aggregates, assembled on the basis of a tractor with an articulated frame, cannot be used to solve this problem. This is mainly because of the atypical layout of the bridge agricultural implement, the specifics of its aggregation and the conditions of operation in the track farming system. In this connection, from the standpoint of efficient application of these bridge agricultural implements with an articulated frame, there are unresolved conditions to be studied that are imposed on their design and other characteristics.

A wide span tractor for the controlled traffic farming system, like any mobile energy tool, is acomplex control object that can be adapted to manual or automatic control and built for the kinematic or power principle of turning (Bulgakov et al., 2020; Gasso et al., 2014). There is a well-known theory of turning the traditional tractor with an articulated frame. However, the dynamics of a flat-parallel movement of the bridge agricultural unit with such a way of turning is not studied. Because of this, at the first stages of research of these bridge agricultural units, the question of studying the dynamics of its flatparallel movement when executing a turn by changing the position of one of its parts relative to the other in a horizontal plane is of scientific interest. It is known from the tractor theory that the operation of a wheeled machine occurs under the action of a large number of disturbing factors (forces and their moments) that change its position in space and deviate its movement from the pre-set path (Bulgakov et al., 2017b). The quality of testing a particular dynamic system of the input variables depends on its characteristics. Concerning the bridge agricultural unit, such are its scheme, as well as the structural and other parameters. Therefore, the right choice of the latter parameters from the position of the necessary controllability and stability of its movement provides the agrotechnical unit with optimal transformation of the controlling and disturbing impacts, acting upon it.
The currently known methodology for the selection of design schemes, parameters and operating modes of the machine-and-tractor aggregates, based on the traditional energy tools with an articulated frame (Farobin, 1970), is practically not suitable for studying the dynamics of the movement of the bridge agricultural units mainly because of their atypical layout scheme, the specifics of aggregation and operating conditions. The structural and technological peculiarities of these bridge agricultural units require the development of a fundamentally new system of their functioning and use.

The analysis conducted by us of the well-known publications (Chamen, 2015; Tullberg, 2013; Pedersen, Oudshoorn \& McPhee, 2016) should state that until now there is no general theory, methodology for calculating the bridge agricultural units and their individual mechanisms, that is, the mechanical and technological foundations of their functioning and use.

The purpose of this study is to establish the degree of influence of the external impacts, as well as structural and technological parameters upon the controllability and stability of movement of a bridge agricultural unit with an articulated frame using a kinematic method of controlling it by turning the semi-frames.

\section{Materials and Methods}

The conducted theoretical studies are based on the use of elements of theoretical mechanics, the theory of mobile energy tools, statistical dynamics and the theory of automatic regulation of linear dynamic systems when they reproduce statistically random control and disturbing input impacts. In particular, the analysis of assessment of the degree of influence of the scheme and parameters of the investigated agricultural tool upon its controllability and stability of movement is based on the amplitude and phase frequency characteristics (Bulgakov, Adamchuk \& Kuvachov, 2017c).

In order to carry out theoretical research, we will present the bridge agricultural unit with the kinematic method of its control by changing the the position of one part relative to the other in a horizontal plane in the form of an equivalent scheme (Figure 1) on which we reflect the forces acting on it.

The external forces, acting upon the bridge agricultural unit during its flat-parallel movement with the kinematic control method by turning the semi-frames, include (Figure 1):

$P_{f 11}, P_{f r 1}, P_{f 2}, P_{f i 2}$ - the resistance forces to rolling of the frontal and rear wheels of the agricultural unit;

$P_{k l 1}, P_{k l 2}, P_{k r 1}, P_{k r 2}$ - the tangential forces;

$R, M_{R}$ - the main vector and the main moment of forces acting from the side of the agricultural implements; 


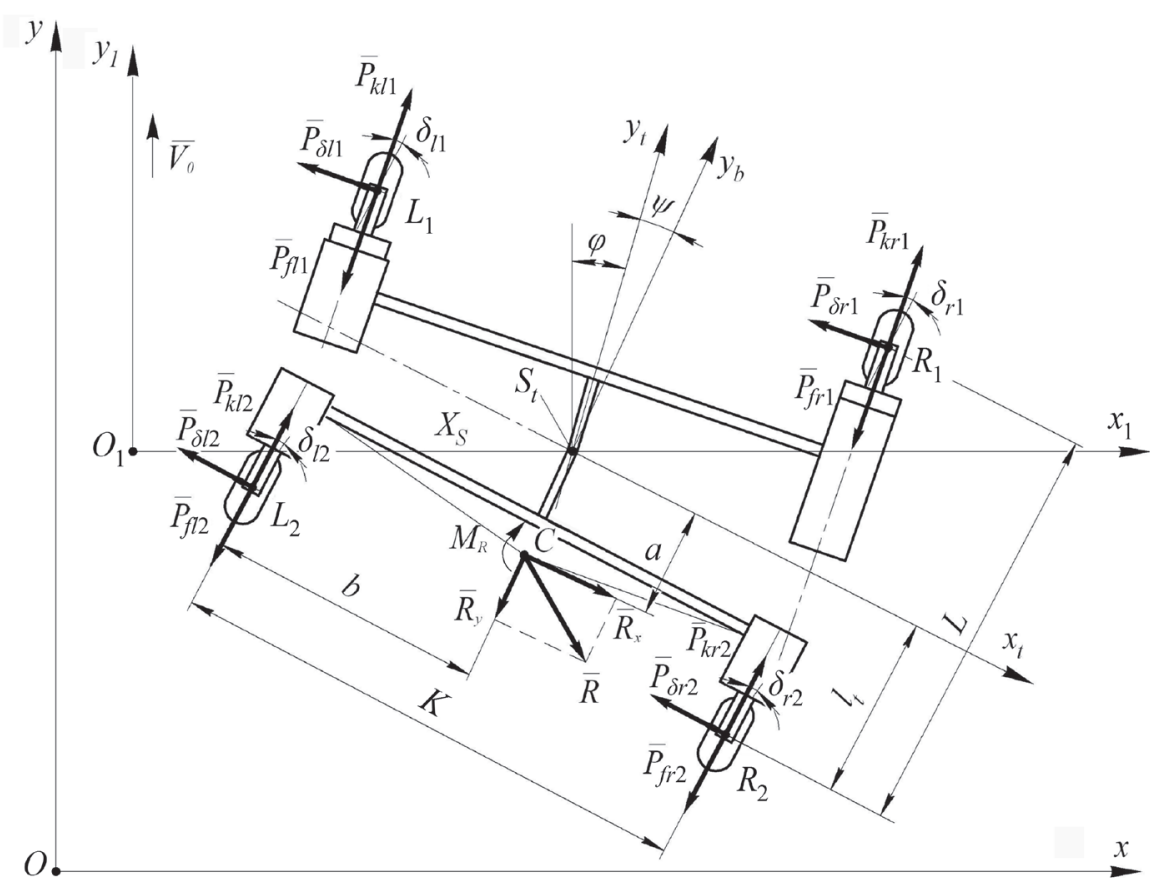

Figure 1. An equivalent scheme of a bridge agricultural unit with its flat-parallel movement in a horizontal plane with a kinematic way of its control by turning the semi-frames.

$P_{\delta l 1}, P_{\delta l 2}, P_{\delta r 1}, P_{\delta r 2}-$ lateral forces, which lead to the appearance of the input angles of the frontal $\delta_{l 1}$, $\delta_{r 1}$ and the rear $\delta_{12}, \delta_{r 2}$ wheels of the agricultural tool.

Differential equations of the movement of the bridge agricultural vehicle on the equivalent circuit (Figure 1) with respect to the plane $x_{1} O y_{1}$ will be compiled in the form of Lagrange equations of the second kind:

$$
\frac{d}{d t}\left(\frac{\partial T_{t}}{\partial \dot{q}_{i}}\right)-\frac{\partial T_{t}}{\partial q_{i}}+\frac{\partial E_{t}}{\partial q_{i}}+\frac{\partial D_{t}}{\partial \dot{q}_{i}}=Q_{i},
$$

where $q_{i}, Q_{i}$ - respectively generalised coordinates and forces;

$T_{t}, E_{t}$ - the kinetic and the potential energy of the bridge agricultural implement;

$D_{t}$ - the dissipative function of energy dissipation by the bridge agricultural implement.

Taking the necessary derivatives with respect to the adopted generalised coordinates, after differentiation (1), a system of differential equations of the following form was obtained:

$$
\left.\begin{array}{l}
M_{t} \cdot \ddot{x}_{s}=Q_{X_{s}}, \\
J_{t} \cdot \ddot{\varphi}=Q_{\varphi},
\end{array}\right\}
$$

where $Q_{X_{s}}$ and $Q_{\ddot{o}}-$ generalised forces that move the bridge agricultural equipment along two generalised coordinates $X_{S}$ and $\ddot{O}$.
As a result of fundamental theoretical research, a mathematical model of the movement of the bridge agricultural unit in a horizontal plane is obtained in a differential form of writing with a kinematic way of its control by turning the semi-frames:

$$
\begin{aligned}
& A_{11} \cdot \ddot{x}_{s}+A_{12} \cdot \dot{x}_{s}+A_{13} \cdot \dot{\varphi}+A_{14} \cdot \varphi=f_{11} \cdot \dot{\psi}+f_{12} \cdot \psi+R_{\mathrm{x}}, \\
& A_{21} \cdot \ddot{\varphi}+A_{22} \cdot \dot{\varphi}+A_{23} \cdot \varphi+A_{24} \cdot \dot{x}_{s}=f_{21} \cdot \dot{\psi}+f_{22} \cdot \psi- \\
& -R_{x} \cdot a+M_{R}-(K-b) \cdot\left(P_{k r 1}-P_{f r 1}+P_{k r 2}-P_{f r 2}\right)+ \\
& +b \cdot\left(P_{k l 1}-P_{f l 1}+P_{k l 2}-P_{f l 2}\right),
\end{aligned}
$$

where

$$
\begin{aligned}
& A_{11}=M_{t} ; \\
& A_{12}=\left(k_{l 1}+k_{l 2}+k_{r 1}+k_{r 2}\right) \cdot V_{o}^{-1} ; \\
& A_{13}=\left[\left(k_{l 1}+k_{r 1}\right) \cdot\left(L-l_{t}\right)-\left(k_{l 2}+k_{r 2}\right) \cdot l_{t}\right] \cdot V_{o}^{-1} ; \\
& A_{14}=-\left(k_{l 1}+k_{l 2}+k_{r 1}+k_{r 2}\right) ; \\
& f_{11}=\left(k_{l 2}+k_{r 2}\right) \cdot l_{t} \cdot V_{o}^{-1} ; \\
& f_{12}=\left(k_{l 2}+k_{r 2}\right)+\left(P_{k l 2}-P_{f l 2}+P_{k r 2}-P_{f r 2}\right)-R_{x} ; \\
& A_{21}=J_{t} ; \\
& A_{22}=\left[\left(k_{l 1}-k_{r 1}\right) \cdot\left(L-l_{t}\right)^{2}+\left(k_{l 2}-k_{r 2}\right) \cdot l_{t}^{2}\right] \cdot V_{o}^{-1} ; \\
& A_{23}=-\left(k_{l 1}+k_{r 1}\right) \cdot\left(L-l_{t}\right)+\left(k_{l 2}+k_{r 2}\right) l_{t} ;
\end{aligned}
$$




$$
\begin{aligned}
& A_{24}=\left[\left(k_{l 1}+k_{r 1}\right) \cdot\left(L-l_{t}\right)-\left(k_{l 2}+k_{r 2}\right) \cdot l_{t}\right] \cdot V_{o}^{-1} ; \\
& f_{21}=-l_{t} \cdot\left(k_{l 2}+k_{r 2}\right) ; \\
& f_{22}=-l_{t}\left[\left(k_{l 2}+k_{r 2}\right)+\left(P_{k l 2}-P_{f l 2}\right)-\left(P_{k r 2}-P_{f r 2}\right)\right]+R_{x} \cdot a .
\end{aligned}
$$

In the equations (2) and (3) $M_{t}, V_{o}$ is the mass $(\mathrm{kg})$ and the speed of the agricultural unit, $\left(\mathrm{m} \cdot \mathrm{s}^{-1}\right)$; $k_{l 1}, k_{l 2}$ and $k_{r 1}, k_{r 2}$ - the resistance coefficients of the wheel displacement, respectively, of the left and the right sides of the agricultural unit, $\mathrm{kN} \cdot \mathrm{rad}^{-1} ; P_{k l 1}$, $P_{k l 2}, P_{k r 1}, P_{k r 2}$ - tangential forces on the wheels of the left and right sides of the agricultural unit $\mathrm{kN} ; P_{f 1}$, $P_{f r 1}$ and $P_{f 22}, P_{f r 2}$ - the rolling resistance forces of the frontal and rear wheels of the agricultural unit; $J_{t}$ the moment of the turning inertia of the agricultural unit in a horizontal plane relative to the vertical axis, passing through point $S_{t}, \mathrm{kN} \times \mathrm{m} \times \mathrm{s}^{2} ; K, L, a, b, l_{t}-$ the design parameters of the bridge agricultural unit, $\mathrm{m}$ ( Figure 1). For this type of bridge agricultural equipment, the ratio of its wheelbase $L$ to the track gauge $K$, as a rule, does not exceed 1 . Therefore, in the process of theoretical research, the equality $L=K$.

The amplitude-frequency characteristics represent the dependence of the modules of the transfer functions of the considered dynamic system upon the frequency $\mathrm{w}$, but the phase-frequency characteristics represent the dependence of the phase shift upon w. By the nature of the transforming properties the considered dynamical system should be classified as a tracking system. It is known from practice that, if at its input, in addition to the control action, there is also a disturbing impact (or obstacle), then the difference (i.e. the difference between the desired and the actual characteristics) is formed from two components. One of them is caused by the fact that the tracking system cannot absolutely accurately reproduce the useful signal of the operator of the control action, and the other is caused by the reaction to the obstacle from the disturbance. Moreover, it often turns out that the desire to reduce the first component leads to an increase in the second, and vice versa. Therefore, the task of synthesis and optimisation is to reach a compromise that will provide the desired operating conditions for the dynamical system investigated by us, and any considered transfer function is the sum of the real $U(\omega)$ and the imaginary $V(\omega)$ of its parts:

$$
W(i \omega)=U(\omega)+i V(\omega)
$$

where $U(\omega)$ and $V(\omega)$ - the real and the imaginary parts of the frequency characteristics of the dynamic system.
The objective functions of the modules of the transfer functions $A(\omega)$ and phase shifts $\Phi(\omega)$ were determined by the following relationships:

$$
\begin{aligned}
& A(\omega)=\sqrt{U^{2}(\omega)+V^{2}(\omega)}, \\
& \Phi(\omega)=\arctan \frac{V(\omega)}{U(\omega)} .
\end{aligned}
$$

In the course of studies of the mathematical model (3), describing the plane-parallel movement of the bridge agricultural implement with an articulated frame in a horizontal plane, it was assumed to determine the degree of impact of the turning frequency of its half-frames (frequency of the control action), as well as the design parameters of the considered dynamic system upon its amplitude and phase frequency specifications. The indicated amplitude and phase frequency characteristics were calculated on a PC using the Mathcad program.

As regards the considered dynamic system of the movement of the bridge agricultural implement in a longitudinal-horizontal plane, the parameters of the control impact and its design parameters were chosen in the process of research on the basis that, for the tracking system in the operating frequency range $\omega$, the ideal (or desired) amplitude-frequency characteristics of the initial values for the control the impact should be close to one, or have a sufficient degree of amplification, and for the disturbing impact it should be equal to zero. In addition, it is desirable that the phase shift in the control impact tends to zero, and in the disturbing one, on the contrary, be as large as possible (tends to infinity).

\section{Results and Discussion}

The characteristics of the angular displacement of the half frames $\psi$ made by the bridge agricultural unit are presented in Figure 2 and Figure 3.

Analysis of the calculated amplitude and phase frequency characteristics (Figure 2 and Figure 3) showed that the controllability of the bridge agricultural unit substantially depends on the width of its track $K$. With increasing this parameter to $9.6 \mathrm{~m}$, which corresponds to the ASA-Lift WS 9600 bridge implement, we have a significant underregulation in the dynamic control system (angular deviation $\psi$ ) (Curve 2, Figure 2). Only at a low frequency of the control impact $\left(\omega=0.5 \mathrm{~s}^{-1}\right)$ does the dynamic system tend to an ideal characteristic. At the same time, at this frequency the bridge agricultural implement with a smaller track width of $3.5 \mathrm{~m}$, which corresponds to an agricultural vehicle, has a gain of the input control impact $A>1$, which contributes to readjustment of the dynamic 
system (Curve 1, Figure 2). In this case, the desired frequency of fluctuations of the angular displacement $\psi$ for the half-frames of the bridge agricultural implement is at the level of $1.5 \mathrm{~s}^{-1}$, at which the value of the gain of the input control impact is $A=1$.

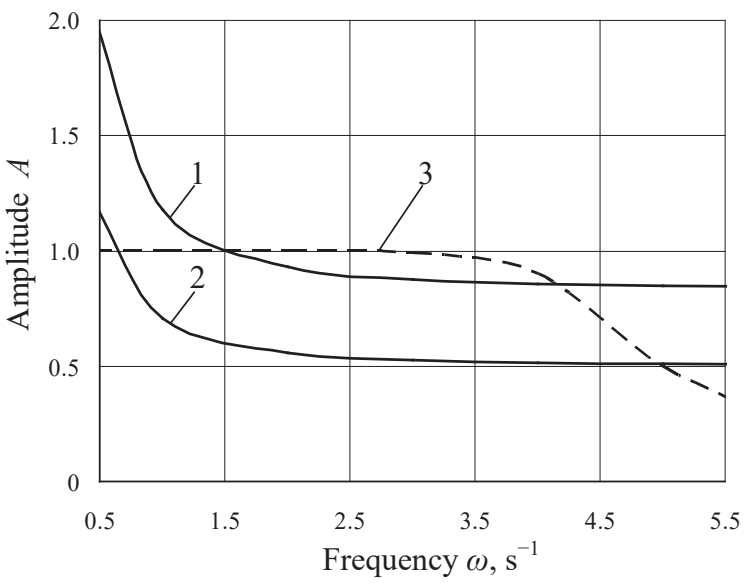

Figure 2. The amplitude $(\mathrm{A}=\varphi / \psi)$ and frequency characteristics of fluctuations in the heading angle $\varphi$ of the agricultural unit performing the control impact (the turning angle $\psi$ of the semi-frames) for various values of its track width: $1-K=3.5 \mathrm{~m}$; $2-K=9.6 \mathrm{~m} ; 3-$ ideal characteristics.

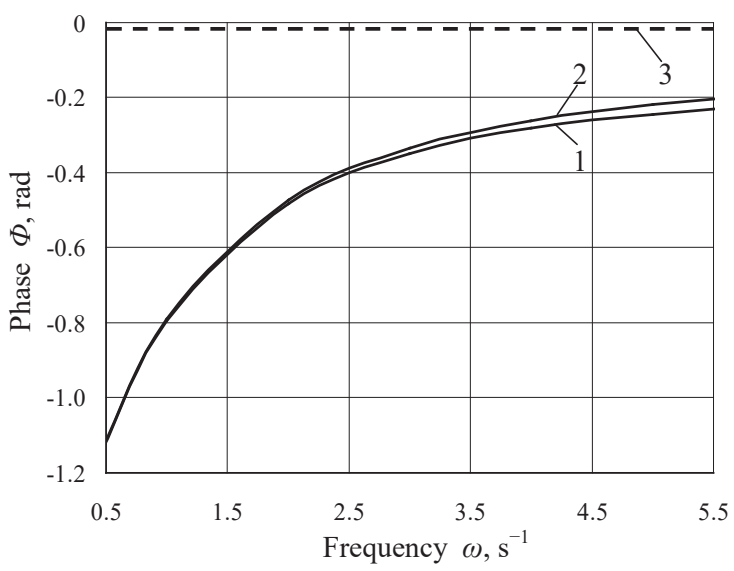

Figure 3. The phase-frequency characteristics of fluctuations in the heading angle $\varphi$ of the bridge agricultural unit when exerting the control impact (the turning-g angle of the half-frames $\psi$ ) with different values of its track width: $1-K=3.5 \mathrm{~m}$; $2-K=9.6 \mathrm{~m} ; 3-$ an ideal characteristic.

However, at low frequencies of the impact the response delay of the dynamic system is as far as possible from the ideal characteristic (Figure 3), and is more than $1 \mathrm{rad}$. And only with an increase in the frequency $\omega$ to $5.5 \mathrm{~s}^{-1}$, the phase-frequency characteristics tend to the ideal. It is worth noting that the increase in the track width of the bridge agricultural implement to $9.6 \mathrm{~m}$, the delay in the response of the dynamic system to the control impact is somewhat less than for the agricultural implement with a track width of $3.5 \mathrm{~m}$ (Figure 3). However, this difference does not exceed $0.05 \mathrm{rad}$.

Consequently, it should be concluded that for the control of the bridge agricultural implement by turning it with half-frames the most efficient option is in which its track width is not large and is within $3.5 \mathrm{~m}$.

The controllability of the agricultural bridge unit with the kinematic method of controlling it by substantially shifting the half-frames depends on the speed of the movement (Figure 4 and Figure 5). At the same time the maximum amplitude of the lateral deviation of the bridge agricultural unit at a low speed regime $\left(V_{o}=1 \mathrm{~m} \cdot \mathrm{s}^{-1}\right)$ in the low-frequency region $\omega$ is at the level of $2 \mathrm{~m}$ per $1 \mathrm{rad}$. of fluctuations of angle $\psi$ (Figure 4).

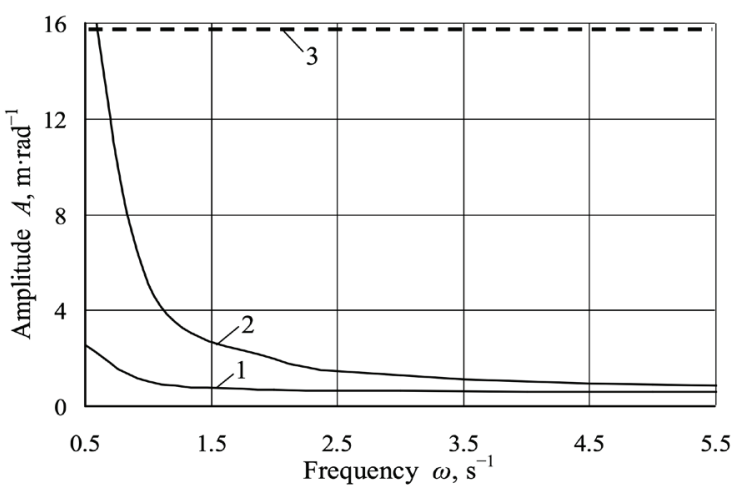

Figure 4. The amplitude-frequency characteristics of the lateral displacement oscillations $X_{s}$ of the bridge agricultural unit when exerting the control impact (the turning angle of the half-frames $\psi$ ) at different speeds of its movement: $1-V_{o}=1 \mathrm{~m} \cdot \mathrm{s}^{-1}$;

$2-V_{o}=3 \mathrm{~m} \cdot \mathrm{s}^{-1} ; 3-$ the desired characteristic.

Increasing the speed regime of the movement of the agricultural unit to $V_{o}=3 \mathrm{~m} \cdot \mathrm{s}^{-1}$, the amplitude of its lateral deviation increases more than $16 \mathrm{~m} \cdot \mathrm{rad} .^{-1}$ (Figure 4), which can practically lead to readjustment of the dynamic system and complexity of control of the agricultural unit, as well as to an increased tramline width by increasing the technological tolerance. In the frequency range of the angle $\psi$ fluctuations, greater than $\omega \geq 2 \mathrm{~s}^{-1}$, the amplitude of the lateral deviation of the agricultural unit decreases and does not exceed $2 \mathrm{~m} \cdot \mathrm{rad}^{-1}$

The delay in the response of the dynamic system to the transverse displacement $X_{s}$ of the bridge agricultural implement when it is developing the control impact (the turning angle of the half frames $\psi$ ) 
practically depends little on the change in frequency $\omega$ in the operating range of $0.5 \ldots 5.5 \mathrm{~s}^{-1}$ (Figure 5). Besides, at a high speed the mode of movement of the bridge agricultural implement $\left(V_{o}=3 \mathrm{~m} \cdot \mathrm{s}^{-1}\right)$, the phase-frequency response is closer to the ideal than at a low speed of its movement. However, this difference does not exceed $0.5 \mathrm{rad}$. On the whole, the phase of the reaction delay of the dynamic system is at the level of $3 \pm 0.5 \mathrm{rad}$ in the range of speeds of movement of the bridge agricultural implement $1 \ldots 3 \mathrm{~m} \cdot \mathrm{s}^{-1}$.

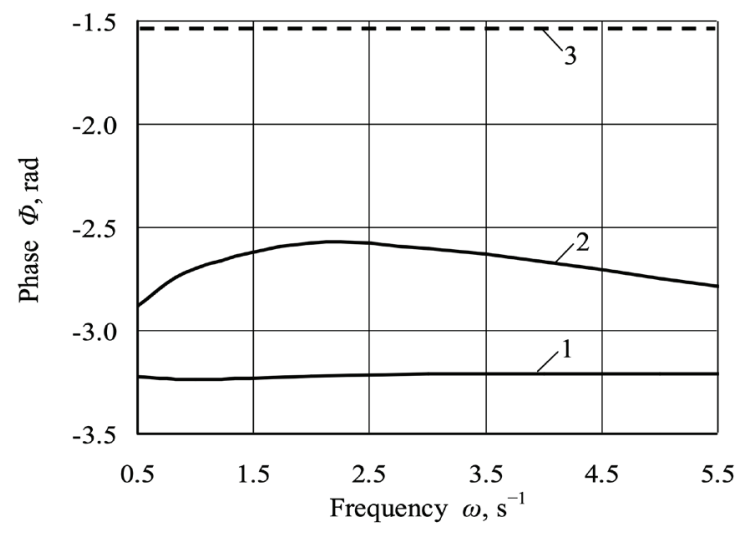

Figure 5. The phase-frequency characteristics of oscillations of the transverse displacement $X_{s}$ of the agricultural unit when exerting the control impact (the turning angle of the half-frames $\psi$ ) at various speeds of its movement: $1-V_{o}=1 \mathrm{~m} \cdot \mathrm{s}^{-1}$;

$2-V_{o}=3 \mathrm{~m} \cdot \mathrm{s}^{-1} ; 3-$ the desired characteristic.

\section{Conclusions}

Mathematical model has been developed, and new regularities of the flat-parallel movement of a wide-span agrocultural unit for the controlled traffic farming system with a kinematic method of its control have been obtained, which allow theoretically to substantiate the new schemas, the design parameters and operating modes with acceptable controllability the movement a horizontal plane.

The nature of the amplitude and phase frequency characteristics substantially depends on the width of the track of the agricultural tool and the speed of its movement. The bridge agricultural implement with a smaller track width of $3.5 \mathrm{~m}$, which corresponds to an agricultural vehicle, has a gain of the input control impact $\mathrm{A}>1$, which contributes to readjustment of the dynamic system. With increasing this parameter to $9.6 \mathrm{~m}$, which corresponds to the ASA-Lift WS 9600 bridge implement, we have a significant underregulation in the dynamic control system. From apposition to ensure better controllability of the bridge agricultural unit with the kinematic method of its control by turning the half-frames, the desired frequency of fluctuations of angle $\psi$ should be at the level of $1.5 \mathrm{~s}^{-1}$

Increasing the speed regime of the movement of the agricultural unit to $V_{o}=3 \mathrm{~m} \cdot \mathrm{s}^{-1}$, the amplitude of its lateral deviation increases more than $16 \mathrm{~m} \cdot \mathrm{rad}^{-1}$, which can practically lead to readjustment of the dynamic system and complexity of control of the agricultural unit, as well as to an increased tramline width by increasing the technological tolerance. In the frequency range of the angle $\psi$ fluctuations, greater than $\omega \geq 2 \mathrm{~s}^{-1}$, the amplitude of the lateral deviation of the agricultural unit decreases and does not exceed $2 \mathrm{~m} \cdot \mathrm{rad}^{-1}$.

\section{References}

Bulgakov, V., Pascuzzi, S., Ivanovs, S., Nadykto, V., Nowak, J., (2020). Kinematic discrepancy between driving wheels evaluated for a modular traction device. Biosystems Engineering, Vol. 196, pp.88-96.

Bulgakov, V., Adamčuk, V., Nozdrovicky, L., Kuvachov, V. (2018). Study of effectiveness of controlled traffic farming system and wide span self-propelled gantry-type machine. Research in Agricultural Engineering. Vol. 64(1), pp. 1-7.

Bulgakov, V., Adamchuk, V., Kuvachov, V., Ivanovs, S. (2017a). Research of possibilities for efficient use of wide span tractor (vehicle) for controlled traffic farming. Proceedings 16 International Scientific Conference «Engineering for Rural Development» (24 - 26 May 2017, Jelgava, Latvia), Vol. 16, pp. 281-287.

Bulgakov, V., Adamchuk, V., Kuvachov, V., Ivanovs, S. (2017b). Theoretical justification of the turn of a wide span tractor (vehicle) for controlled traffic farming. INMATEH - Agricultural Engineering. Vol. 53, (3), pp. 159-167.

Bulgakov, V., Adamchuk, V., Kuvachov, V. (2017c). Study into movement of wide span tractor (vehicles) used in controlled traffic farming. Proceedings $28^{\text {th }}$ DAAAM International Symposium «Intelligent Manufacturing And Automation» (08 - 11th November 2017, Zadar, Croatia) / DAAAM International. Vienna, Austria, pp. 199-208.

Chamen, T. (2015). Controlled traffic farming - From worldwide research to adoption in Europe and its future prospects. Acta Technologica Agriculturae, 18 (3), pp. 64-73.

Farobin A. (1970). The theory of rotation of transport vehicles. Moscow: Mechanical Engineering, 174 p. (In Russian).

Gasso, V., Oudshoorn, F.W., Sørensen, C.A.G., Pedersen, H.H. (2014). An environmental life cycle assessment of controlled traffic farming. Journal of Cleaner Production, 73, pp. 175-182. 
Volodymyr Bulgakov, Semjons Ivanovs, Volodymyr Volskyi, Volodymyr Kuvachov, Yevhen Ihnatiev
Simulation of the Flat-parallel Movement of a Bridge Agricultural Unit with an Articulated Frame
Onal, I. (2012). Controlled traffic farming and wide span tractors. Agricultural Machinery Science, Vol. 8, No 4, pp. 353-364.

Pedersen, H., Oudshoorn, F., McPhee, J. (2016). Wide span re-mechanizing vegetable production. XXIX International Horticultural Congress on horticulture: sustaining lives, livelihoods and landscapes: international symposia on the physiology of perennial fruit crops and production systems and mechanization, precision horticulture and robotics Book Series: Acta Horticulturae. Vol. 1130. pp. 551-557.
Pogorely, L.V. (2008). Сельскохозяйственные машины и технологии будущего (Agricultural machinery and technology of the future). Kiev: Harvest, 176 p. (In Ukrainian)

Tullberg, J. (2013). CTF impacts: Enviroment= Economic. CTF impacts: Environment = Economic. Controlled Traffic Conference. Proceedings ISTRO, Toowoomba, $8 \mathrm{p}$.

Zhalnin, E.V., Mufteyev, R.S. (2002). The history of development and prospects for the introduction of bridge crop production. Tractors and agricultural machinery, No. 5, pp. 23-30. 